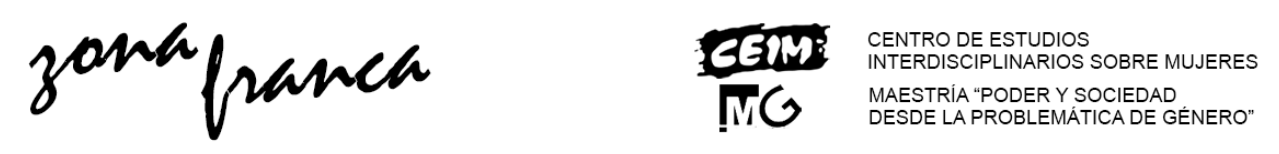

\title{
A 50 años del servicio de aborto feminista de Jane en Chicago
}

Raquel Irene Drovetta*

\section{Resumen}

Este trabajo se propone recuperar, reunir, y analizar documentos sobre la organización de mujeres de Chicago, conocidas como Jane, quienes llevaron adelante un servicio de aborto clandestino, entre 1969 y 1973.

Elaboramos una reconstrucción histórica de los aspectos centrales de la experiencia con el objetivo de mostrar las estrategias que desarrollaron para para implementar el servicio, aun sin contar con instrucción formal en medicina.

Se consultaron archivos conformado por materiales producidos por la organización y entrevistas de ex integrantes del grupo, disponibles en el marco del proyecto de archivo documental HerStory de Chicago Women's Liberation Union. Este procedimiento de trabajo con fuentes fue complementado, por datos secundarios obtenidos a partir de artículos de divulgación, periódicos y filmes, y en publicaciones científicas producidas en las últimas cuatro décadas.

Entre las conclusiones centrales destacamos la centralidad que ocupó para la organización Jane la lucha por el aborto libre y la importancia otorgada al modo de atención de las mujeres basado en una praxis feminista. Remarcamos que se trata de demandas con notable vigencia en el contexto latinoamericano actual.

Palabras clave: aborto - feminismos - organizaciones feministasJane - Chicago Women's Liberation Union

\section{0 years after Jane's feminist abortion service in Chicago.}

\footnotetext{
* Socióloga y doctora en Antropología Social por la Universidad de Buenos Aires. Actualmente se desempeña como investigadora del Consejo Nacional de Investigaciones Científicas y Técnicas y como Profesora Adjunta de la carrera de Sociología de la Universidad Nacional de Villa María. Contacto: raqueldrovetta@conicet.gov.ar
}

Drovetta Raquel. "A 50 años del servicio de aborto feminista de Jane en Chicago" en Zona Franca. Revista del Centro de estudios Interdisciplinario sobre las Mujeres, y de la Maestría poder y sociedad desde la problemática de Género, N²8, 2020 pp. 1-21. ISSN, 2545-6504 Recibido: 1 de agosto 2020; Aceptado: 27 de octubre 2020

Revista Zona Franca- Centro de estudios interdisciplinario sobre las mujeres (CEIM)- Maestría poder y sociedad desde la problemática de género (MG), Rosario, Argentina. ISSN, 2545-6504 http://zonafranca.unr.edu.ar/index.php/ZonaFranca| Número 28 (2020). Página 1 


\section{Abstract}

This paper aims to retrieve, gather, and analyze documents about the Chicago women's organization, known as "Jane", Who carried out a clandestine abortion service between 1969 and 1973.

We elaborate on the historical reconstruction of the central aspects of the experience to show the strategies that They developed to implement the service, even without having formal instruction in medicine.

We consulted archives with materials produced by the organization and interviews with ex-members of the group, which are available in the Chicago Women's Liberation Union's HerStory documentary archive project. We complement this working procedure with sources with secondary data obtained in popular articles, newspapers, and films, and scientific research produced in the last four decades.

Among the principal conclusions, we highlight the centrality that the fight for free abortion occupied for the Jane organization, and the importance that they gave to the model of care for women based on a feminist praxis. We emphasize that these are demands with notable validity in the current Latin American context.

Chicago Women's Liberation Union

Keywords: abortion - feminisms - feminist organizations - Jane Chicago Women's Liberation Union

«Miró la hoja de plástico transparente sobre el colchón, el espéculo y la jeringa. Luego se echó a reír y dijo: ¿Por qué haces esto? Usted no es rica. Con lo que cobran no se puede estar haciendo esto por el dinero. ¿De qué se trata? ¿Son ustedes un montón de mujeres partidarias del feminismo?

¿Eso es todo?»

Judith Arcana (1998:12)

\section{Introducción}

En los últimos 50 años, las leyes que regulan el acceso al aborto legal en los países industrializados han garantizado la prestación del servicio de aborto legal, en el ámbito público y/o el privado. Al mismo tiempo, los grupos antiderechos han promovido la derogación de estas leyes. Por ejemplo, en Estados Unidos la ley que permite el aborto-aprobada en 1973, como resultado del fallo de Roe vs. Wade- continúa vigente, pero condicionada por permanentes intentos de grupos neoconservadores por contrarrestar el alcance de los derechos adquiridos. Esto

Revista Zona Franca- Centro de estudios interdisciplinario sobre las mujeres (CEIM)- Maestría poder y sociedad desde la problemática de género (MG), Rosario, Argentina. ISSN, 2545-6504 http://zonafranca.unr.edu.ar/index.php/ZonaFranca| Número 28 (2020). 
incluye tanto el cierre de clínicas de aborto, como la aprobación de numerosas restricciones legales para el acceso a la práctica (Jones y Jerman 2017; Fuentes y Jerman 2019).

Paradójicamente, la situación en gran parte de Latinoamérica no muestra cambios significativos las últimas cinco décadas. En la mayoría de los países el aborto continúa restringido en base a un modelo de causales. Esto se traduce en bajas posibilidades de un acceso real a la práctica de la interrupción voluntaria del embarazo, algo que suele estar complejizado por la obstaculización que genera la burocracia judicial, las barreras impuestas por sectores conservadores y religiosos, la propia cultura institucional de las organizaciones de salud, y las/los profesionales de salud que se declaran objetoras/es de conciencia. Los cambios más recientes se manifestaron en la legalización del aborto en Uruguay (2012) y la aprobación en Chile (2017) de una ley que garantiza la no punibilidad del aborto en tres causales que anteriormente sostenía la prohibición absoluta, como continúa sucediendo en El Salvador, Nicaragua, República Dominicana y Honduras.

En Argentina, existen recientes avances en dirección al cumplimiento de la interrupción de embarazos en casos amparados por la no punibilidad. Durante 2012 se lograron clarificaciones sobre la aplicación del aborto no punible a partir de la pronunciación de la Corte Suprema sobre el caso F.A.L. y en 2018 se discutió por primera vez en el Congreso de la Nación un proyecto de Ley de Interrupción Voluntaria del Embarazo. Si bien la ley no fue aprobada (el 9 de agosto de 2018 obtuvo 38 votos en contra y 31 a favor), a partir de entonces se aceleraron procesos institucionales conducentes a la creación y adhesión por provincias, de protocolos de atención al aborto no punible en instituciones de salud, entre otros avances. Al mismo tiempo, las organizaciones sociales de base lograron notable adhesión por parte de las mujeres, constituyendo un hito en el movimiento feminista argentino, que incluso mantuvo en vigilia, la noche de la votación, a unos tres millones de personas en la calle.

Revista Zona Franca- Centro de estudios interdisciplinario sobre las mujeres (CEIM)- Maestría poder y sociedad desde la problemática de género (MG), Rosario, Argentina. ISSN, 2545-6504 http://zonafranca.unr.edu.ar/index.php/ZonaFranca| Número 28 (2020). 
En este trabajo, nos interesa recuperar las características centrales del movimiento conocido como "Jane" o "el Servicio" que funcionó en Chicago a inicios de los años 70 , con el objetivo de nutrir una genealogía de prácticas de colectivización feminista, que pueda ser interpretada a la luz de los cambios que atraviesan los movimientos de mujeres en Latinoamérica y el Caribe. En un contexto de emergencia de acciones feministas que promueven el acompañamiento a mujeres que requieren un aborto en un contexto de restricciones legales (Grosso, Trpin y Zurbriggen 2013), creemos que las experiencias de las integrantes de los movimientos de la segunda ola del feminismo pueden ser resignificadas e inspiradoras de nuevas praxis.

Metodológicamente, este trabajo se propone recuperar, reunir y analizar documentos referidos a la organización Jane, que ofreció el servicio de aborto en contextos de clandestinidad, en la ciudad de Chicago, Estados Unidos. El período descripto abarca de 1969 a 1973, y se corresponde con el inicio de sus actividades, extendiéndose hasta la disolución del grupo y la legalización del aborto en ese país.

Elaboramos una reconstrucción histórica de los aspectos centrales que caracterizaron a esta organización, en base al análisis de datos directos obtenidos de diversos fondos documentales. Accedimos al registro escrito de las entrevistas a ex integrantes de la organización Jane, así como imágenes y documentos producidos por el grupo. La mayor parte de los documentos consultados han sido recuperados y traducido del inglés, ya que son escasas las referencias a estos grupos producidas en español. Respecto a la traducción de fuentes, adherimos a postulados de Temple y Young (2004) respecto a los desafíos que esto representa para la investigación cualitativa. Una parte importante de los documentos de la organización fueron digitalizados y se encuentran disponibles en bases de datos especializadas, gracias a organizaciones que promueven el rescate y puesta en valor de experiencias feministas de diversas épocas. Un ejemplo es el archivo

Revista Zona Franca- Centro de estudios interdisciplinario sobre las mujeres (CEIM)- Maestría poder y sociedad desde la problemática de género (MG), Rosario, Argentina. ISSN, 2545-6504 http://zonafranca.unr.edu.ar/index.php/ZonaFranca| Número 28 (2020). 
construido por The Chicago Women's Liberation Union, denominado HerStory Projecty disponible en la Web.

Este procedimiento de trabajo con fuentes fue complementado por datos secundarios obtenidos a partir de publicaciones de divulgación e investigaciones científicas producidas en las últimas cuatro décadas. El conjunto de datos fue analizado a partir de descriptores clave para la temática y según los supuestos que guiaron la investigación, referidos a las prácticas que desarrollaron las activistas de la organización en la atención de abortos clandestinos.

El acceso al material digital fue realizado en la biblioteca de University of Guelph en Ontario, Canadá, en el marco de una estancia postdoctoral realizada por la autora (2017 y 2018) y financiada por una beca del Consejo Nacional de Investigaciones Científicas y Técnicas (CONICET).

\section{Antecedentes: organizaciones feministas que realizan abortos}

Con anterioridad a las manifestaciones de carácter organizado y numeroso promovidas por el feminismo llamado de la segunda ola, se ha documentado (aunque de manera escasa) la existencia de otra organización que trabajó para cambiar las restrictivas leyes de aborto estadounidenses, aunque con una estructura de funcionamiento diferente. Se trata de The Society for Humane Abortion (SHA) fundada por Patricia Magginis en California en 1965, quien se uniría a Rowena Gurner y a Lana Phelan, a quienes se conocería como el Ejército de tres (Army of Three) (Reagan, 2000). Algunas autoras consideran a estas mujeres como las primeras activistas por el derecho al aborto en la historia de Estados Unidos (Reagan, 1997; Baehr, 1990).

Las prácticas de sus antecesoras difieren a las de Jane, pero constituyen parte ineludible de la historia de cómo los derechos sexuales y reproductivos han estado en el centro de la preocupación de las feministas. El aspecto que hace a

Revista Zona Franca- Centro de estudios interdisciplinario sobre las mujeres (CEIM)- Maestría poder y sociedad desde la problemática de género (MG), Rosario, Argentina. ISSN, 2545-6504 http://zonafranca.unr.edu.ar/index.php/ZonaFranca| Número 28 (2020). 
Jane una organización particular es que ofreció durante varios años un servicio de aborto clandestino, prescindiendo de médicos titulados y cuyas actividades estuvieron organizadas en torno a una praxis feminista de la atención a la interrupción del embarazo.

En la revisión de antecedentes para otro trabajo (Drovetta, 2020), encontramos experiencias similares para la época. Estas han sido referidas en trabajos de investigación de carácter histórico, como por ejemplo el movimiento italiano de mujeres y feministas y su vinculación con el Partido Radical para llevar adelante la práctica de abortos clandestinos (documentado por Bracke, 2015 y 2017; Cilumbriello y Colombo, 2001, entre otras). También en Europa el movimiento de izquierda y feminista de Francia es descripto en trabajos de investigación a partir de la construcción de una red de apoyo de grupos de mujeres que realizaban abortos clandestinos en Francia (Pavard, 2009 y 2012; Zancarini-Fournel, 2003). Estas dos experiencias europeas coinciden, entre otras cosas, en mostrar cómo la coalición entre partidos políticos de izquierda, organizaciones de mujeres y en menor medida, profesionales de la salud, oficiaron en un doble plano: en la práctica realizaban abortos seguros a mujeres que cursaban embarazos no planificados mientras que, en el plano político, litigaban, generaban lobby y se mostraban en las calles y en espacios públicos representativos, demandando leyes que permitieran acceder al aborto libre. El hilo conductor que conecta estas experiencias es que, dentro de esta trama de actores, existieron organizaciones integradas por mujeres legas en medicina, que practicaban abortos clandestinos en diferentes países. Posiblemente este sea uno de los escasos puntos en común entre las organizaciones nombradas, pero constituye el nodo central desde donde partir.

\section{Movimientos de Mujeres de Chicago (CWLU) y el servicio "Call} Jane"

Revista Zona Franca- Centro de estudios interdisciplinario sobre las mujeres (CEIM)- Maestría poder y sociedad desde la problemática de género (MG), Rosario, Argentina. ISSN, 2545-6504 http://zonafranca.unr.edu.ar/index.php/ZonaFrancal Número 28 (2020). 
La experiencia del servicio que ofreció Jane a las mujeres de Chicago continuó silenciada hasta que a fines de los '80 paulatinamente emergieron producciones documentando la experiencia a partir de testimonios ofrecidos por ex integrantes del servicio (inicialmente Bart, 1987; Kaplan, 1997; Riddiough, 2015; Strobel y Davenport, 1999; Surgal, 1999; Jepson, 1999; Galatzer-Levy, 2014; Schmid y Riddlough, s/d). Posiblemente la publicación más reveladora sea el libro donde Laura Kaplan, una ex integrante de la organización, describe detalladamente la experiencia. Se trata de The story of Jane: The legendary underground feminist abortion service, en sus ediciones de 1995, 1997 y 2019. Mientras tanto, otros trabajos de investigación reconstruyen la historia en base a testimonios indirectos para dar a conocer la experiencia (Baehr, 1990; King, 1993; Reagan, 1997; Weisman, 1998; Morgen, 2002; Griffin 2015; O'Donnell, 2017).Numerosos testimonios de ex integrantes del servicio de aborto han sido publicados en el sitio https://www.cwluherstory.org/ que oficia como archivo de documentos que describen el papel desarrollado por Chicago Women's Liberation Union entre 1969 y 1977.

Las investigaciones en castellano son muy escasas, entre ellas destaca la investigación sobre organizaciones feministas desarrollada por Mabel Bellucci (2014). La divulgación de la existencia de esta organización a nivel local despertó el interés de otras agrupaciones, dando como resultado el trabajo prologado e impulsado por la editorial feminista chilena Dejemos la Escoba, que traduce del inglés, en 2014, Jane: documentos del servicio clandestino de aborto de Chicago (1968-1973),una compilación que incluye entrevistas y textos producidos por antiguas participantes del grupo(originalmente titulado Jane: documents from Chicago's clandestine abortion service, 1968-1973) y la traducción al español de Gabriela Adelsteinen (2013) de un texto publicado en una compilación de Marlene Gerber Fried (1990). Las producciones nombradas ponen de manifiesto el interés de feministas latinoamericanas por dar a conocer la propuesta traduciendo al español literatura generada por el activismo norteamericano.

Revista Zona Franca- Centro de estudios interdisciplinario sobre las mujeres (CEIM)- Maestría poder y sociedad desde la problemática de género (MG), Rosario, Argentina. ISSN, 2545-6504 http://zonafranca.unr.edu.ar/index.php/ZonaFranca| Número 28 (2020). 
La historia de la organización también se recrea a través de diversos productos culturales. La historia de Jane fue ganando visibilidad como la obra de teatro Jane: Abortion and the Underground (1993) de Paula Kamen y del documental de Kate Kirtz y Nell Lundy, Jane: An Abortion Service (1996) entre los principales.'

\section{Los inicios de la organización}

La organización Chicago Women's Liberation Union (Unión de Liberación de las Mujeres de Chicago) conocida por sus siglas CWLU, se conformó como la mayor organización socialista-feminista que se estableció a fines de los años '60 en Estados Unidos.

Casi la totalidad de sus integrantes eran mujeres blancas, pertenecientes a la clase media, con estudios universitarios, quienes creían en la igualdad de las mujeres y entendían que esto sólo podía lograrse combatiendo al racismo, al clasismo y al sexismo al mismo tiempo (Griffin, 2015; Jepson, 1999; Riddiough, 2015). Quienes formaron parte del grupo también adherían a los movimientos antibélicos y antiimperialistas y al movimiento por los derechos civiles (Riddiough, $\mathrm{s} / \mathrm{d})$.

La organización CWLU, operó con una estrategia fundada en tres pilares: el servicio, la educación y la acción directa. Desde esta perspectiva se concentró en atender a numerosos intereses que incluyeron la salud de las mujeres y los derechos reproductivos, la educación, los derechos económicos, las artes visuales y la música, los deportes, la liberación de las lesbianas, la justicia económica, los derechos de las reclusas y la oposición a la guerra; (Strobel y Davenport, 1999; Riddiough, 2015; Schmid y Riddlough, s/d; Bellucci, 2014).En la práctica, CWLU funcionó como una estructura paraguas bajo la que confluyeron una amplia gama de proyectos de trabajo, de discusión y de concientización, y donde las activistas

I Información disponible en https://www.paulakamen.com/ Acceso 12 de febrero de 2018.

Revista Zona Franca- Centro de estudios interdisciplinario sobre las mujeres (CEIM)- Maestría poder y sociedad desde la problemática de género (MG), Rosario, Argentina. ISSN, 2545-6504 http://zonafranca.unr.edu.ar/index.php/ZonaFranca| Número 28 (2020). 
circularon peticiones, realizaron manifestaciones y visitaron escuelas secundarias para aumentar la conciencia pública sobre los problemas de la mujer (Strobel y Davenport, 1999).

En 1969 integrantes de esta organización inician un servicio para mujeres que requirieran un aborto, que hará historia. Formalmente llamado Abortion Counseling Service of Women's Liberation, será conocido como Jane o el Servicio. Durante los cuatro años que estuvo en marcha la experiencia, más de cien mujeres fueron integrantes de Jane y se calcula que se realizaron alrededor de once mil abortos (Kaplan, 1997).

Algunas investigaciones y documentos de la época sostienen que la relación entre el colectivo Jane y CWLU era de afiliación, lo que implicaba una total autonomía del movimiento y que inclusive, Jane podría haber sido creada 5 años antes que CWLU (Jepson, 1999; Hyde Park-Kenwood Voices, 1973). Por ejemplo Leslie Reagan(1997)ubica el año de inicio de Jane en 1967, a partir de la iniciativa de Heather Booth, estudiante de la Universidad de Chicago, quien comenzó a ofrecer referencias sobre médicos confiables que realizaran abortos, mientras que el periódico alternativo Hyde Park, en relación con la creación de CWLU, advierte que "si bien decidimos no afiliarnos oficialmente a la Unión, trabajamos en estrecha colaboración, organizando grupos de apoyo y trabajando para cambiar la opinión pública sobre el aborto, mientras trabajábamos para que el aborto estuviera disponible"(Hyde Park-Kenwood Voices, 1973: s/d).

La experiencia de Chicago coincide con el surgimiento en Estados Unidos de un conjunto de organizaciones de mujeres congregadas en torno a demandas relacionadas con la atención a la salud sexual y reproductiva de las mujeres desde un paradigma diferente a la propuesta médica hegemónica (Morgen, 2002; Weisman, 1998; Baehr, 1990). Entre estas experiencias destaca The Boston Women's Health Collective (Colectivo de salud de las mujeres de Boston) quienes presentaron en 1970 su icónica publicación Our Bodies Ourselves. Este libro se

Revista Zona Franca- Centro de estudios interdisciplinario sobre las mujeres (CEIM)- Maestría poder y sociedad desde la problemática de género (MG), Rosario, Argentina. ISSN, 2545-6504 http://zonafranca.unr.edu.ar/index.php/ZonaFranca| Número 28 (2020). 
convirtió en un referente del feminismo estadounidense ( $y$ posteriormente internacional), al poner en palabras el presupuesto sobre el que se asentaron otras organizaciones feministas contemporáneas: las mujeres se sentían descontentas con "el establecimiento médico capitalista y patriarcal" (Boston Women's Health Book Collective, 1998:136) al cuál debían recurrían para atender su salud y enfermedad (Musser, 2007; Wells, 2008; Davis, 2007; Hayden, 1997).

Al mismo tiempo avanzaban fundamentalmente en California los grupos de activismo político como los de concientización (consciousnessraising) (Morales, 2019) y otros más específicos como los de help-self. Este último se caracterizaba por la conformación de espacios donde era posible desplegar un conjunto de prácticas que permitían a las mujeres explorar su cuerpo y su sexualidad, basándose en la idea de que la liberación política debía estar arraigada en una nueva relación con el cuerpo, lo que implicaba un desafío explícito a la medicina establecida, así como el objetivo de articular formas alternativas de conocimiento (Bracke, 2015).

Comprensiblemente, la salud se consolidó como un área clave en CWLU que combinó educación, servicio y acción directa de las activistas.

\section{Jane en funcionamiento}

Inicialmente, se trató de un servicio clandestino de consejería sobre el aborto, a través del cual un grupo de mujeres favorecía el contacto entre quienes buscaban un aborto y los médicos prestadores del servicio. La conformación era heterogénea e incluía a estudiantes, amas de casa y algunas profesionales. Mientras que la organización central de CWLU definía la membresía al grupo a través de la adhesión al conjunto de principios ideológicos definidos anteriormente, ninguna identidad feminista era necesaria para involucrarse en Jane (Bart, 1987; Griffin, 2015). La organización ofrecía en un panfleto: "somos mujeres cuyo objetivo final es la liberación de las mujeres en la sociedad. Una forma importante

Revista Zona Franca- Centro de estudios interdisciplinario sobre las mujeres (CEIM)- Maestría poder y sociedad desde la problemática de género (MG), Rosario, Argentina. ISSN, 2545-6504 http://zonafranca.unr.edu.ar/index.php/ZonaFrancal Número 28 (2020). 
en la que estamos trabajando para lograr ese objetivo es ayudar a cualquier mujer que desee un aborto a obtener uno de la manera más segura y económica posible bajo las condiciones existentes" (Jane panfleto, 1969-1973)."

Las ex integrantes de la organización relatan cómo el contacto entre ellas y las mujeres se realizaba a través del llamado a un número telefónico donde atendía una activista cuyo nombre ficticio siempre era Jane. A partir del contacto se iniciaba la búsqueda de un aborto en la clandestinidad, lo que equivalía a obtener un aborto en condiciones "cuidadas" durante la intervención. Esto incluía que, observando ciertas precauciones, las mujeres no se verían expuestas a la policía.

El aborto debía pagarse, aun cuando en ocasiones por la intermediación de las integrantes de Jane frente al médico, se lograban descuentos en aquellos casos en que las mujeres no pudieran reunir todo el dinero. Las intervenciones se realizaban en hoteles, bajo notables medidas de seguridad tendientes fundamentalmente a mantener el anonimato y la seguridad de los médicos.

En el transcurso de los primeros meses de funcionamiento, las responsabilidades de las mujeres en el servicio evolucionaron más allá de la consejería y el otorgamiento de referencias sobre donde abortar (las "pasadoras")."I Ellas comenzaron a ofrecer asistencia técnica al médico durante la intervención, se familiarizaron con la técnica de dilatación y curetaje y eventualmente recibieron instrucciones sobre cómo realizar los procedimientos. De esta manera, el aprendizaje se convirtió en parte del componente político del aborto para las activistas involucradas. Como sostiene Reagan (1997) Jane era tanto un servicio de salud como un proyecto de educación política.

\footnotetext{
"Traducción propia, citado en https://www.paulakamen.com/jane-play-and-resources/ Acceso 2 de noviembre de 2018.

III Grosso, Trpin y Zurbriggen (2013) definen la tarea de "pasadoras" en una organización feministas actual.
}

Revista Zona Franca- Centro de estudios interdisciplinario sobre las mujeres (CEIM)- Maestría poder y sociedad desde la problemática de género (MG), Rosario, Argentina. ISSN, 2545-6504 http://zonafranca.unr.edu.ar/index.php/ZonaFrancal Número 28 (2020). 
Tiempo después, integrantes de Jane descubren que en realidad el médico no era un profesional de la salud titulado, lo que finalmente las animó a prescindir de él y realizar ellas mismas las intervenciones. En el invierno de 1971, las activistas comenzaron a realizar todo el procedimiento, constituyéndose como proveedoras del servicio de aborto a cualquier mujer, en cualquier instancia del embarazo y aun cuando no pudieran pagar (Bart, 1987). También, tomaron en sus manos la decisión acerca de donde se realizarían los abortos, ofreciendo sus propias casas o rentando apartamentos temporalmente (Kaplan, 1997).

A esta altura, la organización era autosustentable, lo que permitió mayor dedicación de las activistas al servicio, evitando destinar energías en la búsqueda de fuentes externas de sostenimientos (Bart, 1987). El funcionamiento del grupo se complejizó y las integrantes de Jane debieron distribuirse roles como retomar el contacto con las mujeres que habían llamado al teléfono y dejado sus datos, ubicar un espacio para realizar los abortos, ordenar a las mujeres que llegaban en gran número a la cita. Una vez allí, debían ofrecer conserjería e informar en detalle sobre lo que acontecería y lo que sentiría durante el procedimiento, para finalmente, aplicar la técnica del aborto y realizar las tareas conexas como el manejo y cuidado del instrumental, la compra de drogas e insumos para las intervenciones, entre otras actividades (Kaplan, 1997; Bart, 1987).

El precio promedio de un aborto se redujo de más de u\$400 a menos de u\$100. Aun así, muchas mujeres no podían pagar esa cifra, especialmente después de 1973, cuando el aborto se legaliza en el Estado de Nueva York y las mujeres de clase media acudían a las clínicas en ese Estado. A partir de entonces, el sistema evidenció sus limitaciones ya que las mujeres que acudían a Jane eran las más pobres y de color (Bart, 1987; Galatzer-Levy, Jeanne; Proyecto Herstory, 2014; Surgal, 1999).

\section{La praxis feminista del aborto}

Revista Zona Franca- Centro de estudios interdisciplinario sobre las mujeres (CEIM)- Maestría poder y sociedad desde la problemática de género (MG), Rosario, Argentina. ISSN, 2545-6504 http://zonafranca.unr.edu.ar/index.php/ZonaFranca| Número 28 (2020).

Página 12 
En sus memorias, integrantes del servicio reflexionan cómo "claramente, aprender a hacer un DyC [acrónimo de procedimiento dilatación y curetaje] nosotras mismas, de principio a fin, fue el último paso esencial para tener un servicio que pudiera ser controlado y administrado por mujeres" (Hyde ParkKenwood Voices, 1973: s/d).

En el espacio de Jane se animaba a las mujeres a hablar de sí mismas y sus experiencias personales en relación con el aborto, el embarazo, los hijos, entre otros temas (Surgal, 1999). Cuando las mujeres se encontraban relajadas y en compañía, el aborto era un procedimiento menos incómodo (Kaplan, 1997). Incluso, durante las consejerías y sesiones informativas, las activistas remarcaban a las mujeres su poder de agencia y decisión (Griffin, 2015) y relatando lo que sucedía durante el procedimiento a pacientes lúcidas, también las involucraban en el procedimiento. Estas prácticas dejaban en evidencia la notable diferencia con el rol de paciente estipulado en la práctica médica convencional.

La instalación del servicio de aborto significó poder conciliar la teoría y la práctica, al proporcionar un modelo concreto de acción, en línea con una visión revolucionaria más amplia, permitiendo a las mujeres tomar la salud en sus manos, sin recurrir a servicios lucrativos y dominados por hombres. De la misma forma, al enfatizar el alto costo del aborto ilegal, enmarcaron la referencia al servicio como un tema de justicia económica y feminista.

\section{Ausencia de redes feministas}

Jane puede ser descripto como un grupo con limitado contacto con otras organizaciones feministas. Su base de apoyo parece haber estado constituida por personal farmacéutico y de la salud, que en determinadas instancias ofició como vínculo para obtener insumos médicos e información. Algunas fuentes refieren a la tarea de varones cercanos a las integrantes (parejas) quienes por ejemplo se encargaban de deshacerse de residuos patógenos de manera segura.

Revista Zona Franca- Centro de estudios interdisciplinario sobre las mujeres (CEIM)- Maestría poder y sociedad desde la problemática de género (MG), Rosario, Argentina. ISSN, 2545-6504 http://zonafranca.unr.edu.ar/index.php/ZonaFranca| Número 28 (2020). 
Sin embargo, existe un aspecto central que muestra los efectos resultantes de la no circulación de determinada información entre redes, y es el hecho de que las activistas de Jane continuaron utilizando la técnica de dilatación y curetaje, cuando en California otros grupos utilizaban ya la técnica de aspiración perfeccionada por Karman. Tanfer Tunc detalla en sus investigaciones cómo la práctica, actualmente conocida como de aspiración manual al vacío, comenzó a ser desarrollada en Estados Unidos a partir de la década del '50 cuando la perfeccionó Harvey Karman, un psicólogo y activista de California (Tunc, 2008; 2008a). La utilización del método de aspiración permitió reducir las complicaciones postabortos, redujo la necesidad de anestesia y habilitó la posibilidad de optar por la participación de las mujeres durante el procedimiento.

\section{La clandestinidad, la ilegalidad y la relación con la policía.}

El sentido otorgado a la noción de clandestinidad debe ser discutido con detenimiento para cada uno de los contextos en que el aborto es analizado. En este caso, el aborto no era legal al momento de la creación de la organización de Chicago y el servicio seguiría activo hasta la legalización en todos los Estados Unidos, en 1973. Sin embargo, la detención y encarcelamiento de sus integrantes fue un evento que contribuyó a acelerar el proceso de desintegración de la organización.

La relación entre la organización Jane y la policía local siempre había sido de no interferencia. Las suposiciones indican que era conveniente parala fuerza de seguridad, no molestar a la organización que atendía los abortos de esposas, hijas, amantes de policías y las mujeres policías también (Bart, 1987).

Sin embargo, luego de años sin ninguna detención ni intromisión del personal policial, el 3 de mayo de 1972 la policía de Chicago se presentó en uno de los departamentos donde integrantes de Jane estaban realizando abortos y detuvo a activistas y otras personas presentes en el lugar, quienes quedaron libres bajo

Revista Zona Franca- Centro de estudios interdisciplinario sobre las mujeres (CEIM)- Maestría poder y sociedad desde la problemática de género (MG), Rosario, Argentina. ISSN, 2545-6504 http://zonafranca.unr.edu.ar/index.php/ZonaFrancal Número 28 (2020). 
fianza el día siguiente. Mientras se desarrolló lo que se llamó el "caso aborto 7" en el que siete integrantes de Jane fueron acusadas de conspirar para cometer abortos, esperaron el resultado en libertad y al menos cuatro de ellas volvieron a trabajar al servicio (Galatzer-Levy, 2014). La estrategia de la defensa fue dilatar la presentación ante el juez, esperando que la corte se expidiera sobre el caso Roe vs. Wae, lo que sucedió el año siguiente. El contexto de ebullición social en el que se asomaba la legalización del aborto en Estados Unidos fue favorable para lograr la eximición de la pena, y las acusaciones finalmente se disolvieron y el caso cayó.

Cuando el aborto se declara legal en 1973, comenzaron a abrir las clínicas. La permanencia o no en la tarea de la prestación del servicio de aborto después de la legalización, fue un tema discutido en el interior del grupo. El objeto central del debate era analizar la necesidad o validez acerca de que un servicio con características específicas como el ofrecido por Jane, debería o no continuar funcionando en la clandestinidad. Si trabajaban ocultándose del Estado, en un contexto donde la interrupción del embarazo era legal y el servicio se ofrecía en instituciones de salud, funcionaría como un servicio paralelo y no autorizado. Sin embargo, el leitmotiv que fundaba la existencia de los grupos se basaba en el convencimiento de que las feministas prestaban un servicio diferente, improbable de ser replicado por médicos en las instituciones de salud. Consecuentemente, el nuevo contexto legal habilitaba a que el poder estuviera concentrado exclusivamente en manos de los médicos, lo que significaría ofrecer atención a la salud, prescindiendo de una perspectiva de cuidado como la que había instituido el colectivo Jane. La praxis feminista del aborto quedaría fuera de los consultorios.

Al analizar las argumentaciones, se evidencian los intereses políticos que constituyeron la base de las acciones realizadas. Es necesario entonces pensar que la práctica del aborto que llevaron adelante las estadounidenses contribuyó a diversos objetivos. El principal, fue ofrecer una opción confiable a las mujeres que deseaban interrumpir un embarazo no planeado. Al mismo tiempo, la práctica del aborto en manos de activistas feministas buscó contrarrestar una realidad aún

Revista Zona Franca- Centro de estudios interdisciplinario sobre las mujeres (CEIM)- Maestría poder y sociedad desde la problemática de género (MG), Rosario, Argentina. ISSN, 2545-6504 http://zonafranca.unr.edu.ar/index.php/ZonaFranca| Número 28 (2020). 
vigente: el Estado no está presente y las mujeres mueren como consecuencia de abortos clandestinos.

Cuando finalmente el grupo se disuelve, parte de las integrantes continuaron participando de otros proyectos de acción feministas vinculados fundamentalmente con la defensa de los derechos sexuales y reproductivos. Por ejemplo, después de que se legalizó el aborto se formó el Servicio de Educación y Referencia de Salud de CWLU para guiar a las mujeres a clínicas seguras y respetuosas (Jepson, 1999).

\section{Conclusiones}

¿Que representa Jane en la actualidad? Como sostiene Kelly O’Donnell, las integrantes de Jane y las simpatizantes de la segunda ola feministas, pueden apoyarse en la historia de la organización como forma de mantener la relevancia de los mensajes políticos en tiempos de profundos cambios (2017: 93). Recordar esta historia ofrece varias oportunidades: otorgar significado político a la práctica del aborto permite construir un significado superador de la simple práctica médica, que podría resultar despolitizador. Al mismo tiempo, creemos que el rescate de esta experiencia contribuye a ofrecer expectativas respecto a la fortaleza de las acciones colectivas de las mujeres, que han demostrado ser capaces de generar movimientos underground cuyo efecto,50 años después, aún continúa generando diversas sensaciones. Esto remite a la eficacia de la memoria y los efectos que esta tiene sobre el presente.

Por otra parte, visto a la distancia, la cuantificación de los hechos contribuye a otorgar materialidad a los eventos: 11.000 abortos, 100 activistas, cuatro años de servicios. La repetición de estas características hace posible alimentar la mística de una épica que fue posible, en condiciones tan o más complejas que las actuales y que aun así se sostuvo en el tiempo. Jane ha sido convertida en un icono de tipo inspirador de las nuevas generaciones. Finalmente, también

Revista Zona Franca- Centro de estudios interdisciplinario sobre las mujeres (CEIM)- Maestría poder y sociedad desde la problemática de género (MG), Rosario, Argentina. ISSN, 2545-6504 http://zonafranca.unr.edu.ar/index.php/ZonaFrancal Número 28 (2020). 
podemos coincidir con Bibia Pavard quien sostiene en referencia a las organizaciones de Francia: "la práctica de los abortos ofrece una fuerte atracción. La recompensa simbólica inmediata que proporciona ayudar a las mujeres, al igual que violar la ley, pueden traer gran satisfacción" (2012:53).

\section{Bibliografía}

"Jane" (1990). «Just Call "Jane"» En From Abortion to Reproductive Freedom: Transforming a Movement, en Marlene GERBER FRIED, (ed.). Traducción de Gabriela ADELSTEIN, pp. 93-100. Boston: South End Press. Disponible en www.rimaweb.com.ar Último acceso: 22 de enero de 2015.

ARCANA, Judith (1998). «Ella dijo antes de 1973» CALYX 17, Revista de Arte $y$ Literatura hecha por Mujeres, $\mathrm{N}^{\circ} 3$, invierno.

ARCANA, Judith, GALATZER-LEVY Jeanne, y SURGAL Ruth (2004). Jane: documents from Chicago's clandestine abortion service, 1968-1973.Chicago Women's Liberation Union Herstory Project, Archivo histórico, disponible enhttps://www.cwluherstory.org/ Baltimore: Firestarter Press, p. 58.

BAEHR, Ninia (1990). Abortion Without Apology. A Radical history for the 1990s.Boston: South End Press.

BART, Pauline (1987). "Seizing the means of reproduction: An illegal feminist abortion collective-How and why it worked» En Qualitative Sociology 10 (4): 339357.

BART, Pauline, ROSENRobyn L., y O’DONNELL Pauline (2014). «Panel "A Revolutionary Moment"» Boston University. 29th de March. Disponible en https://youtu.be/FvpSQOtrTz0 Ultimo acceso: 1 de agosto de 2020.

BELLUCCI, Mabel (2014). Historia de una desobediencia: aborto $y$ feminismo. Ciudad Autónoma de Buenos Aires: Capital Intelectual.

BOSTON WOMEN'S HEALTH BOOK COLLECTIVE (1998). Our bodies, ourselves for the new century: $A$ book by and for women. Boston: Touchstone Books.

Revista Zona Franca- Centro de estudios interdisciplinario sobre las mujeres (CEIM)- Maestría poder y sociedad desde la problemática de género (MG), Rosario, Argentina. ISSN, 2545-6504 http://zonafranca.unr.edu.ar/index.php/ZonaFranca| Número 28 (2020). 
BRACKE, Maud A. (2017). «Feminism, the state, and the centrality of reproduction: abortion struggles in 1970s Italy» En Social History 42 (4): 524-546, https://doi.org/10.1080/03071022.2017.1368234

BRACKE, Maud Anne (2015). "Our Bodies, Ourselves: The Transnational Connections of 1970s Italian and Roman Feminism» En Journal of Contemporary History, 50 (3): 560-580, https://doi.org/10.1177/0022009414552869

CILUMBRIELLO, Antonietta, y COLOMBO Daniela (2001). "The Fight for Reproductive Rights in Italy» En Advocating for Abortion Access: Eleven Country Studies, KLUGMAN Barbara y BUDLENDER Debbie (eds.) pp. 157-172. Johannesburg: Women's Health Project, School of Public Health, University of the Witwatersrand.

DAVIS, Kathy (2007). The making of our bodies, ourselves: How feminism travels across borders. Duke: Duke University Press.

DROVETTA Raquel I. (2020). «La práctica del aborto en manos de feministas en los 70». En Revista Estudos Feministas, (en prensa).

FIRESTARTER PRESS (2014). Jane: documentos del servicio clandestino de aborto de Chicago (1968-1973). Traducido por Dejemos La Escoba. Santiago de Chile: Dejemos La Escoba.

FUENTES, Liza, y JERMAN Jenna (2019). «Distance Traveled to Obtain Clinical Abortion Care in the United States and Reasons for Clinic Choice» En Journal of Women's Health, December, 16231631,https://doi.org/10.1089/jwh.2018.7496

GALATZER-LEVY, Jeanne (2014). «En el trabajo con Jane» En FIRESTARTER PRESS, Jane - Documentos del servicio clandestino de aborto de Chicago (1968 -1973), traducido por Editorial "Dejemos la Escoba", pp. 33-46. Santiago de Chile: Dejemos La Escoba.

GRIFFIN, Lara (2015). "The Chicago Women's Liberation Union: White Socialist Feminism and Women's Health Organizing in the 1970s» Thesis, BA, History, Oberlin College. Electronic Thesisor Dissertation. Disponible en https://etd.ohiolink.edu/ Ultimo acceso: 10 de agosto de 2018.

Revista Zona Franca- Centro de estudios interdisciplinario sobre las mujeres (CEIM)- Maestría poder y sociedad desde la problemática de género (MG), Rosario, Argentina. ISSN, 2545-6504 http://zonafranca.unr.edu.ar/index.php/ZonaFrancal Número 28 (2020). 
GROSSO, María B., TRPIN María, y ZURBRIGGEN Ruth (2013). «Políticas de y con los cuerpos: cartografiando los itinerarios de Socorro Rosa (un servicio de acompañamiento feminista para mujeres que deciden abortar)" En Género y diversidades sexuales: devenires, deseos y derechos; FERNÁNDEZ Ana M. SIQUEIRA PERES Wilson. Buenos Aires: Biblos.

HAYDEN, Sara (1997). «Re-claiming bodies of knowledge: An exploration of the relationship between feminist theorizing and feminine style in the rhetoric of the Boston women's health book collective» En Western Journal of Communication 61 (2): 127-163.

HYDE PARK-KENWOOD VOICES (1973). «The Most Remarkable Story Ever Told» Chicago Women's Liberation Union Herstory Project, Archivo histórico, disponible en https://www.cwluherstory.org/ Último acceso: 12 de mayo de 2020.

JEPSON, Cara (1999). Sister Against the System. The Chicago Reader, periódico. 5 de marzo de 1999. Disponible en https://www.chicagoreader.com/chicago/sisters-against-thesystem/Content?oid=898587 Último acceso: 10 de mayo de 2020.

JONES, Rachel K., y JERMAN Jenna (2017). «Abortion Incidence and Service Availability In the United States, 2014» En Perspect Sex Reprod Health 49 (1): 17-27, https://doi.org/10.1363/psrh.12015

KAPLAN, Laura (1997). The story of Jane: The legendary underground feminist abortion service. Chicago: University of Chicago Press.

KING, Charles R. (1993). «Calling Jane» En Women \& Health,20 (3): 75-93, https://doi.org/10.1300/J013v20n03 05

MORALES, Gabriela M. (2020). Mujeres que acompañan a mujeres. Experiencias, sentidos y prácticas de Socorro Rosa Córdoba. Tesis presentada para acceder al grado de Magister en Intervención e Investigación Psicosocial, Facultad de Ciencias Sociales, Universidad Nacional de Córdoba, Inédita.

MORGEN, Sandra (2002). Into Our Own Hands. The women's health movement in the United States, 1969-1990.New Brunswick: Rutgers University Press.

Revista Zona Franca- Centro de estudios interdisciplinario sobre las mujeres (CEIM)- Maestría poder y sociedad desde la problemática de género (MG), Rosario, Argentina. ISSN, 2545-6504 http://zonafranca.unr.edu.ar/index.php/ZonaFranca| Número 28 (2020). 
MUSSER, Amber J. (2007). «From Our Body to Yourselves: The Boston Women's Health Book Collective and Changing Notions of Subjectivity 1969-1973» En Women's Studies Quarterly, 35 (1/2): 93-109.

O'DONNELL, Kelly S. (2017). "Reproducing Jane: Abortion Stories and Women's Political Histories» En Signs: Journal of Women in Culture and Society, 43 (1): 77-96, https://doi.org/10.1086/692444

PAVARD, Bibia (2009). "Genre et militantismedans le Mouvement pour la liberté de l'avortement et de la contraception. Pratique des avortements (19731979)» En Clio. Histoire, femmes et sociétés,29: 7996, https://doi.org/10.4000/clio.9217

PAVARD, Bibia (2012). «Quand la pratique fait mouvement. La méthode Karmandans les mobilisations pour l'avortement libre et gratuit (1972-1975)» En Sociétés contemporaines, 85 (1): 43-63, https://doi.org/10.3917/soco.085.0043

REAGAN, Leslie J. (2000). "Crossing the border for abortions: California activists, Mexican clinics, and the creation of a feminist health agency in the 1960s» En Feminist Studies, 26 (2): 323-348.

REAGAN, Leslie J. (1997). When abortion was a crime: Women, medicine, and law in the United States, 1867-1973.California: Univ. of California Press.

RIDDIOUGH, Chistine. s/d. Health Care Organizing in the Chicago Women's Liberation Union. Chicago Women's Liberation Union Herstory Project, Archivo histórico. Disponible en https://www.cwluherstory.org/analysis-articles/health-careorganizing-in-the-chicago-womens-liberation-unionúltimo acceso: 10 de marzo de 2020.

RIDDIOUGH, Christine (2015). Our Band of Sisters. Building Culture and Community in the Chicago Women's Liberation Union. Chicago Women's Liberation Union Herstory Project, Archivo histórico. Disponible en https://www.cwluherstory.org/our-band-of-sisters/ Último acceso: 18 de agosto de 2018.

SCHMID, Margaret, y RIDDLOUGH Chris (2016). Socialist Feminist Theory \& Strategy in the Chicago Women's Liberation Union. Chicago Women's Liberation

Revista Zona Franca- Centro de estudios interdisciplinario sobre las mujeres (CEIM)- Maestría poder y sociedad desde la problemática de género (MG), Rosario, Argentina. ISSN, 2545-6504 http://zonafranca.unr.edu.ar/index.php/ZonaFranca| Número 28 (2020). 
Union Herstory Project, Archivo histórico. Disponible en https://www.cwluherstory.org/theory-strategy Ultimo Acceso: 10 de mayo de 2020.

STROBEL, Margareth, y DAVENPORT Sue (1999). «The Chicago Women's Liberation Union: An Introduction by Margaret "Peg" Strobel and Sue Davenport» Chicago Women's Liberation Union Herstory Project, Archivo histórico. Disponible en https://www.cwluherstory.org Último acceso: 8 de agosto de 2018.

SURGAL Ruth (1999). Organizing a Clandestine Abortion Network.Chicago Women's Liberation Union Herstory Project, Archivo histórico. Disponible enhttps://www.cwluherstory.org/jane-stories-articles/organizing-a-clandestineabortion-network?rq=Jane\%3A\%20 Último acceso: 10 de agosto de 2018.

TEMPLE, Bogusia, y YOUNG Alys (2004). "Qualitative Research and Translation Dilemmas» En Qualitative Research, 4 (2): 161-178. https://doi.org/10.1177/1468794104044430

TUNC, Tanfer E. (2008). «Designs of devices: The vacuum aspirator and American abortion technology» En Dynamis 28: 353-376.

TUNC, Tanfer E. (2008a). "Harvey Karman and the super coil fiasco: A forgotten episode in the history of abortion technology» En European Journal of Contraception and Reproductive Health Care, 13 (1): 4-8. https://doi.org/10.1080/13625180701746461

WEISMAN, Carol S. (1998). Women's Health Care. Activist Traditions and Institucional Change. Baltimore: The Johns Hopkins University Press.

WELLS, Susan (2008). "Our bodies, ourselves: Reading the written body» En Signs: Journal of Women in Culture and Society 33 (3): 697-723.

ZANCARINI-FOURNEL, Michelle (2003). «Histoire(s) du MLAC (1973-1975)» En Clio. Histoire, femmes et sociétés. 18, https://doi.org/10.4000/clio.624

Revista Zona Franca- Centro de estudios interdisciplinario sobre las mujeres (CEIM)- Maestría poder y sociedad desde la problemática de género (MG), Rosario, Argentina. ISSN, 2545-6504 http://zonafranca.unr.edu.ar/index.php/ZonaFrancal Número 28 (2020). 